\title{
Apports d'éléments minéraux au sol par l'intermédiaire de la litière, des pluies et des pluviolessivats dans un peuplement à genévrier thurifère (Juniperus thurifera L) du Haut Atlas occidental (Maroc)
}

\author{
T Gauquelin 1, F Fromard 2, W Badri 3, J Dagnac 4 \\ 1 Université $P$ Sabatier, laboratoire de botanique et biogéographie, \\ 39, allées Jules-Guesde, 31062 Toulouse Cedex et CRSE, UPR CNRS 9014; \\ 2 Institut de la Carte Internationale de la Végétation, UPS, UA CNRS 688, \\ 39, allées Jules-Guesde, 31062 Toulouse Cedex; \\ 3 Université Cadi Ayyad, faculté des sciences, laboratoire d'écologie végétale, \\ BP S15 Marrakech, Maroc; \\ 4 UPS, laboratoire de botanique et biogéographie, 39, allées Jules-Guesde,
} 31062 Toulouse Cedex et UA CNRS 695

(Reçu le 28 février 1992; accepté le 3 septembre 1992)

\begin{abstract}
Résumé - Différents paramètres du cycle biogéochimique ont été étudiés pendant 2 ans dans une formation ouverte à genévrier thurifère (Juniperus thurifera $L$ ) située à $2450 \mathrm{~m}$ d'altitude dans le Haut Atlas occidental au Maroc. La quantité d'éléments nutritifs apportée au sol par les pluviolessivats et la litière sous la couronne des arbres est élevée (42 g. $\mathrm{m}^{-2}$ de couvert.an $\left.{ }^{-1}\right)$. Les apports par la pluie et les pluviolessivats fortement enrichis en éléments minéraux $(\times 3,8)$ constituent $20 \%$ du total. $80 \%$ sont donc apportés par la litière dont la production moyenne est importante $\left(1100 \mathrm{~g}^{-\mathrm{m}^{-2}}\right.$ de couvert.an ${ }^{-1}$ ) et qui est particulièrement riche en calcium. Ces différents apports, strictement localisés sous le couvert des arbres participent grandement à l'hétérogénéité édaphique de ces milieux ouverts.
\end{abstract}

Juniperus thurifera L / cycle biogéochimique / pluviolessivat / forêt présteppique / Haut Atlas marocain

Summary - Contribution of minerals to the soil via litter, rainfall and throughfall in a juniper tree population (Juniperus thurifera $\mathrm{L}$ ) in the western High Atlas (Morocco). The amount of nutrient reaching the soil below the crown of a juniper tree (Juniperus thurifera $L$ ) located at an altitude of $2450 \mathrm{~m}$ in the western High Atlas in Morocco and showing a characteristic "open structure" was estimated from 1987 to 1989. The amount of nutrient returned to the soil below the tree crown was high (42 g.year-1. $\mathrm{m}^{-2}$ for the macroelements). Input from rainfall, throughfall and stemflow constituted $20 \%$ of this amount and a clear increase was noted in the nutrient concentration in throughfall 
compared to rainfall $(x 3.8)$. The high input from litter ( $80 \%$ of the total input) was linked to the high mean annual litterfall (1 $100 \mathrm{~g} \cdot \mathrm{m}^{-2}$ of crown coverage) and to the particularly high concentration of calcium in the litter.

Juniperus thurifera L / biogeochemical cycle / throughfall / pre-steppe forest / Moroccan High Atlas

\section{INTRODUCTION}

Le cycle des éléments minéraux dans les écosystèmes forestiers et en particulier leur apport au sol par l'intermédiaire des pluies, pluviolessivats et de la litière a souvent été étudié en région méditerranéenne française (Lossaint et Rapp, 1980) et, sous même type de climat, au Liban (Dannaoui, 1981), en Espagne (Escudero et al, 1985; Santa Regina et Gallardo, 1989) ou en Tunisie (Selmi, 1985). Dans les milieux altimontains de ces mêmes régions, $a$ fortiori en Afrique du Nord, les recherches restent peu développées. Les rares données existant dans le domaine du fonctionnement des écosystèmes forestiers d'altitude au Maroc concernent essentiellement les bilans hydriques (Lepoutre, 1961).

Nos travaux portent sur les communautés à Genévrier thurifère (Juniperus thurifera L) qui constituent souvent dans le Haut Atlas marocain la limite forestière supérieure et montrent des caractéristiques structurales et édaphiques tout à fait particulières (Gauquelin 1988, 1989). Ces forêts présteppiques (Barbero et Quézel, 1984) sont caractérisées par une structure ouverte - les couronnes des arbres sont rarement jointives - et par un microédaphisme strictement dépendant du couvert arboré (Gauquelin et Dagnac, 1988). Une ambiance forestière marquée par une stabilité édaphique et une pédogenèse active règne ainsi sous le couvert des arbres alors qu'entre ceux-ci prédominent les processus morphogénétiques caractéristiques des milieux supraforestiers méditerranéens.
La surexploitation du bois par les habitants des hautes vallées atlasiques vient encore accentuer la précarité de ce milieu, fragilisé déjà par une absence quasi générale de régénération et une croissance très lente des genévriers (Fromard et Gauquelin, 1992).

L'évolution des éléments minéraux entre les différents compartiments de l'écosystème - feuillage, litière, sol - a été suivie pendant 2 années afin de mettre en évidence le rôle particulier du thurifère dans la différenciation édaphique observée. La composition des eaux de pluie et des pluviolessivats, dans une atmosphère a priori non polluée chimiquement mais souvent chargée en pous. sières, et dans des conditions tout à fait particulières, méritait également d'être étudiée.

\section{MATÉRIELS ET MÉTHODES}

\section{Caractéristiques du site d'étude}

La thuriféraie étudiée occupe le versant sudouest du Jbel Tizrag $\left(31^{\circ} 13^{\prime} \mathrm{N}, 7^{\circ} 53^{\prime} \mathrm{W}\right.$, massif de l'Oukaimeden, Haut Atlas occidental), entre $2200 \mathrm{~m}$ et $2600 \mathrm{~m}$ d'altitude. Le peuplement, constitué d'arbres adultes mais aussi de quelques jeunes, est peu dégradé. Sous les genévriers, de nombreuses germinations de cette espèce apparaissent régulièrement, ce qui est assez rare dans ce type de formation au Maroc.

Le thurifère, qui domine dans les strates arborée et arbustive, est accompagné d'un autre genévrier, Juniperus oxycedrus. La strate chaméphytique est occupée par les xérophytes épineuses en coussinets Bupleurum spinosum, Cytisus purgans var balansae et, aux expositions nord et en limites altitudinales supérieures, Ptilo- 
trichum spinosum. Les autres espèces notables de la formation sont Daphne laureola et llex aquifolium, indicateur de faciès plus humide, Stipa nitens, abondant tout au long du versant, Genista florida et Ribes uva crispa plus rares. Le sex-ratio du thurifère (espèce dioïque) calculé dans cette forêt sur un échantillon de 200 individus adultes montrent une répartition entre sexes équilibrée : 104 individus mâles pour 96 individus femelles.

La parcelle mise en défens $\left(1000 \mathrm{~m}^{2}\right)$ occupe un replat à $2450 \mathrm{~m}$ d'altitude où la densité des arbres adultes s'élève à 190/ha, supérieure à ce que l'on trouve habituellement dans ce type de milieu. Chaque individu compte le plus souvent 3 troncs distincts. La hauteur moyenne des arbres est de $4,50 \mathrm{~m}$, chacun d'eux détermine au sol un recouvrement moyen (projection de la couronne) de $25 \mathrm{~m}^{2}$. Leur surface terrière est estimée à $74,4 \mathrm{~m}^{2} / \mathrm{ha}$.

Le sol est profond $(0,80$ à $1 \mathrm{~m})$, limoneux argileux en surface à limoneux sableux en profondeur, développé sur grès rouges du Permotrias localement intercalés de bancs calcaires. Sous le couvert des genévriers la litière est abondante, constituant un horizon Ao holorganique épais (10-15 cm; $\mathrm{C} / \mathrm{N}: 20,7 ; \mathrm{pH}: 7,3)$. Le $\mathrm{pH}$, voisin de la neutralité dans les horizons humifères de surface s'acidifie sensiblement en profondeur $(5,8)$.

Les caractéristiques bioclimatiques du site d'étude sont connues grâce aux données recueillies pendant 8 ans (1982-1989) sur le plateau de l'Oukaimeden situé à proximité immédiate (Gauquelin, 1988; Badri, 1990).

La température moyenne annuelle définie sur cette période est de $8,6^{\circ} \mathrm{C}$ (moyenne des maxima : $12,9^{\circ} \mathrm{C}$; moyenne des minima : $4,2^{\circ} \mathrm{C}$ ). Juillet est le mois le plus chaud (moyenne des maxima : $22,4^{\circ} \mathrm{C}$ ), avec également la plus grande amplitude moyenne $\left(9,5^{\circ} \mathrm{C}\right)$, janvier est le mois le plus froid (moyenne des minima = $2,9^{\circ} \mathrm{C}$ ). Les températures extrêmes enregistrées ont été $28^{\circ} \mathrm{C}$ et $-14,5^{\circ} \mathrm{C}$. Le nombre moyen de jour de gel par an est de 126 et 4 mois présentent une moyenne de minima négative (décembre, janvier, février, mars).

L'humidité moyenne maximale est de $77 \%$, variant de $67 \%$ (décembre) à $87 \%$ (mai), les minima se situant entre 33 et $41 \%$ (moyenne annuelle : $37 \%$ ). Juillet est le mois le plus sec avec une moyenne des minima égale à $33 \%$.

Les précipitations annuelles calculées pour les 8 années de mesures sont de 575 mm (590 mm pour la période 1950-1968), caractérisées par une grande variabilité : $0 \mathrm{~mm}$ en avril 1987 et $138 \mathrm{~mm}$ en avril 1989; $85 \mathrm{~mm}$ en décembre 1985 et $20 \mathrm{~mm}$ en décembre 1988. Entre décembre et fin mars, les précipitations sont surtout neigeuses, la neige elle-mème pouvant persister au sol jusqu'à la fin du mois d'avril (1989). La saison sèche $(P<2 T)$ dure 4 mois (juin à septembre) alors qu'elle est de 8 mois à Marrakech (mars à octobre).

La station ainsi définie se situe, selon le climagramme d'Emberger, dans l'ètage bioclimatique subhumide à hiver froid, avec un quotient pluviométrique Q2 égal à 80,3 . Ceci confirme les observations de nombreux auteurs (Peyre, 1975; Achaal et al, 1980), qui ont montré que la thuriféraie marocaine n'était pas limitée au seul bioclimat semi-aride à hiver froid (Emberger, 1939; Quézel, 1957).

\section{Collecte et analyses de litière et de rameaux verts}

Les apports moyens mensuels de litière ont été évalués dans la parcelle entre novembre 1986 et août 1989. Sous un thurifère ont été mis en place 6 pièges circulaires d'une surface unitaire de $0,5 \mathrm{~m}^{2}$. La surface totale de collecte est ainsi de $3 \mathrm{~m}^{2}$, pour une surface de projection de la couronne de $37 \mathrm{~m}^{2}$. La litière est recueillie 1-2 fois par mois et divisée en 3 lots :

- jeunes rameaux de faible diamètre, recouverts de feuilles squamiformes étroitement imbriquées,

- galbules (l'arbre équipé est un arbre femelle),

- "divers", regroupant des rameaux de plus fort diamètre, des fragments d'écorce, de la gomme séchée, etc.

L'ensemble est séché à l'étuve $\left(80^{\circ} \mathrm{C}\right)$ pendant $36 \mathrm{~h}$ puis pesé.

La composition minérale de la litière a été suivie pendant 2 années. Ses différentes fractions sont broyées jusqu'à obtention d'une poudre fine et homogène. Les éléments $\mathrm{Ca}, \mathrm{Mg}$, $\mathrm{K}, \mathrm{Na}, \mathrm{Mn}, \mathrm{Fe}$ et $\mathrm{P}$ sont dosés selon la méthode décrite par Morard et Gullo (1970) : prédigestion nitrique puis calcination d'une prise d'essai de $0,2 \mathrm{~g}$, double attaque à l'acide chlorhydrique, reprise des résidus en extrait aqueux de $100 \mathrm{ml}$. Le dosage est réalisé par spectrophotométrie d'absorption atomique et, pour le phosphore, par colorimétrie (chaîne automatique Techni- 
con). L'azote total est analysé selon la méthode Kjeldahl sur une prise d'essai de $0,15 \mathrm{~g}$, après attaque à l'acide sulfurique concentré.

Parallèlement à la collecte et l'analyse de litière, des rameaux verts ont été prélevés sur la parcelle tous les 2 mois, sur un même lot d'arbres comprenant des pieds mâles et de pieds femelles. Les échantillons ont été analysés comme décrit ci-dessus pour la litière.

\section{Collecte et analyse des eaux de pluie et des pluviolessivats}

Les premières expérimentations ont mis en évidence la forte hétérogénéité des égouttements sous la couronne d'un même arbre, en fonction de l'emplacement des capteurs (distance au tronc, orientation) (Gauquelin, 1988). Le dispositif suivant, rendant compte de ces variations spatiales, a donc été adopté : 12 pluviomètres en plastique ordinaire (volumes d'égouttement) et 6 en polyéthylène (analyses chimiques des eaux), tous recouverts d'un filet protecteur, sont disposés sous l'arbre équipé de pièges à litière, selon 4 orientations et en cercles concentriques, pour recueillir les eaux d'égouttement (fig 1).

Les eaux d'écoulement sont collectées au moyen de gouttières mises en place autour des 4 troncs du même arbre et recueillies dans une bonbonne en plastique. Les pluies incidentes sont recueillies dans 8 pluviomètres placés hors de l'influence du couvert.

Les échantillons d'eau ont été collectés entre septembre 1987 et août 1989, le plus rapidement possible après l'événement pluvieux (délai moyen : $6 \mathrm{j}$ ), afin de limiter au maximum l'évolution chimique des échantillons, leur pollution par des débris divers ou encore l'incorporation des dépôts secs.

Après mesure du $\mathrm{pH}$ et stockage immédiat au congélateur, le dosage des cations ( $\mathrm{Na}, \mathrm{K}$, $\mathrm{Mg}, \mathrm{Ca}, \mathrm{Mn}$ ) est réalisé par spectrophotométrie d'absorption atomique sur échantillons non fil-
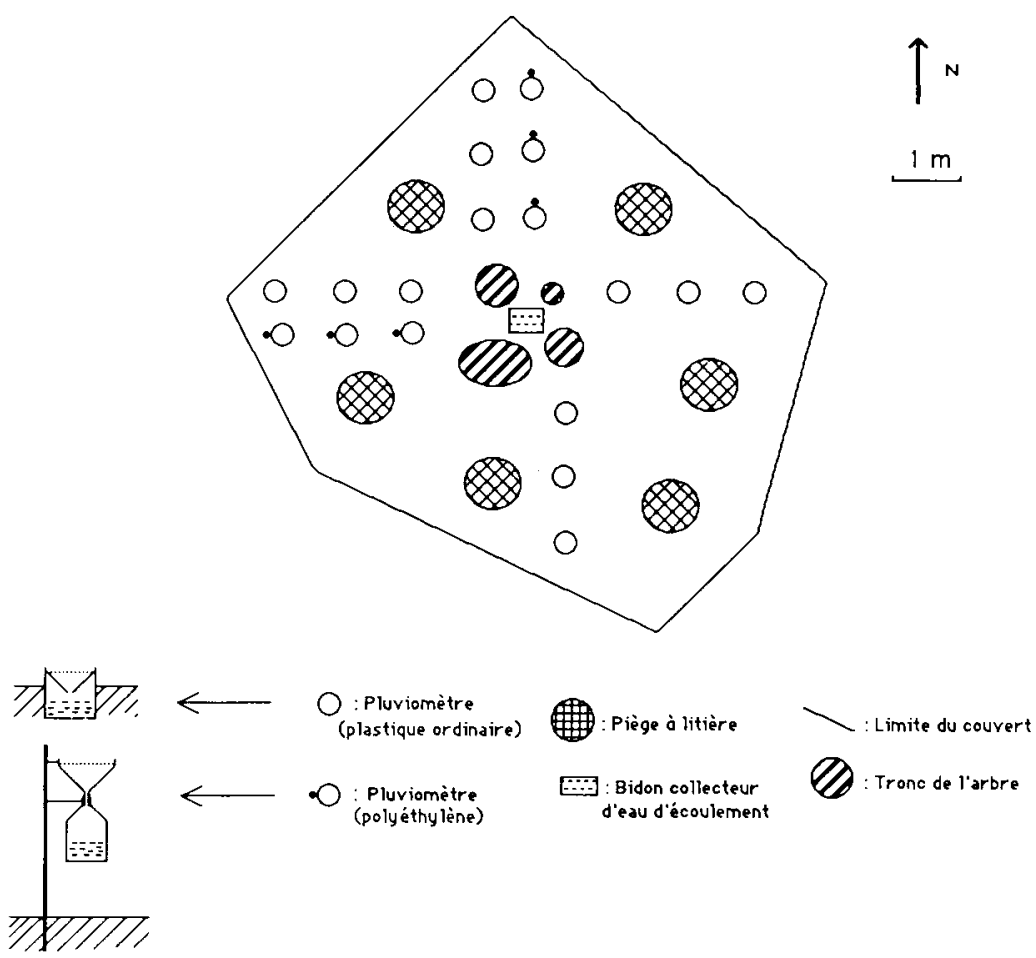

Fig 1. Schéma du dispositif expérimental de récolte de litière et de pluviolessivats sous le couvert d'un genévrier thurifère (parcelle de l'Oukaimeden, Haut Atlas, Maroc). 
trés, après décantation des dépôts particulaires observés dans certains pluviolessivats (des essais préliminaires ont montré qu'il n'existait pas de différence significative entre les résultats pour les échantillons filtrés ou non filtrés). L'azote ammoniacal est analysé sur chaîne automatique Technicon. Les anions majeurs $(\mathrm{Cl}$, $\mathrm{NO}_{3}, \mathrm{SO}_{4}, \mathrm{~F}$ et $\mathrm{P}$ ) sont dosés, après filtration, par chromatographie ionique (HPLC Dionex).

Les flux d'éléments minéraux sont calculés en multipliant, pour chaque événement pluvieux, la concentration moyenne établie à partir de tous les pluviomètres hors ou sous couvert (les eaux récoltées dans les pluviomètres situés à même distance du tronc sont mélangées) par la hauteur moyenne d'eau mesurée dans ces pluviomètres.

L'étude entreprise étant destinée avant tout à mettre en évidence le rôle particulier de l'arbre dans la différenciation édaphique, un seul individu représentatif de la parcelle a été équipé, permettant ainsi une meilleure approche de la variabilité et de l'hétérogénéité des différents apports au sol.

Les résultats obtenus, qui sont les premiers disponibles pour ce type de milieu, sont comparés à ceux de la littérature.

\section{RÉSULTATS}

\section{Restitution des éléments minéraux au sol par l'intermédiaire de la litière}

\section{Analyse quantitative des retombées de litière}

\section{Production annuelle de litière}

Calculée sur les 2 années complètes de collecte dans la parcelle (septembre 1987août 1989), la production moyenne annuelle de litière s'élève à $1100 \mathrm{~g} \cdot \mathrm{m}^{-2}$ de couvert (soit 11 t.ha $^{-1}$ ), avec une variation importante liée à la durée de la saison sèche ainsi qu'à des accidents climatiques locaux (orages, tempêtes) : 650 g.m-2 pour les 12 premiers mois de mesure (septembre 1987 - août 1988) et $1570 \mathrm{~g} \cdot \mathrm{m}^{-2}$ pour les 12 mois suivants (septembre 1988 - août 1989).
Cette production annuelle élevée ramenée à l'ensemble de la surface au sol équivaut à 5,3 t.ha- $\mathbf{a n}^{-1} \cdot \mathrm{an}^{-1}$ (la projection au sol des couronnes dans la parcelle représentant $48 \%$ de la surface totale). Ces valeurs sont alors comparables à celle donnée par Aussenac et al (1972)

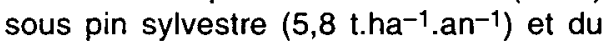
même ordre de grandeur que celle établie par Selmi (1985) pour le chêne zeen en Tunisie $\left(4,8\right.$ t.ha $\left.^{-1} \cdot a n^{-1}\right)$. Elles sont de plus en accord avec le diagramme de retombées de litière de Bray et Gorham (1964) qui indique pour la latitude de l'Oukaimeden $\left(31^{\circ} \mathrm{N}\right)$, une moyenne annuelle de 6 t.ha- ${ }^{-1} \cdot$ an $^{-1}$.

D'autres travaux (Aussenac et al, 1972 et 1979) font état de valeurs plus faibles sur l'épicéa $\left(3,8\right.$ t.ha- $\left.^{-1} . \mathrm{an}^{-1}\right)$ ou le douglas (3,5 t.ha-1.an-1). De même Léonardi et al (1988) pour les peuplements de pin laricio de l'Etna donnent 2,8 t.ha- $^{-1}$.an-1. Une production annuelle plus importante (7,8 tha${ }^{1}$.an-1) apparaît par contre chez Pinus pinea en région méditerranéenne française (Cabannetes, 1979).

Chez le thurifère, la fraction jeunes rameaux + feuilles, avec 620 g.m ${ }^{-2}$.an ${ }^{-1}$, constitue $56 \%$ de la litière totale, avec une variation saisonnière importante $(40 \%$ en novembre 1988 à $70 \%$ en août 1989). Ce résultat est comparable au pourcentage de feuilles participant à la litière du chêne vert dans les dehesas du Sud de l'Espagne (Escudero, 1985), mais plus faible que celui donné pour le pin parasol $(75 \%)$ par Dannaoui (1981).

La fraction galbule représente $25,5 \%$ de la litière totale, témoignant de la fructification abondante de cet arbre, et la fraction «divers» $18,5 \%$.

En tout état de cause, l'écosystème thuriféraie apparaît donc, à surface de couvert égale, tout aussi productif que d'autres formations forestières méditerranéennes ou médio-atlantiques. 


\section{Rythme des retombées}

La figure 2 met en évidence la rythmicité des chutes de litière, avec un pic principal entre août et octobre, aussi bien en 1988 qu'en 1989. Cette période de retombées maximales est plus tardive que celle signalée par Rapp (1967) pour le pin d'Alep (juillet), mais synchrone de celle donnée par Dannaoui (1981) pour le pin parasol. En 1987, ces valeurs maxima étaient beaucoup moins élevées, en liaison probablement avec la sécheresse du début d'année qui a limité la croissance des arbres et la production foliaire. Un phénomène semblable a été observé par Rapp (1975) sur le pin d'Alep.

En période hivernale, des chutes importantes peuvent être aussi notées, provoquées par de violentes tempêtes (janvier 1987).

Enfin, ponctuellement, l'importance de la fraction "petits rameaux" qui est le plus souvent dominante, peut diminuer : en octobre 1988 la fraction galbules a égalé cette dernière et, en juin 1989, la fraction "divers" a dépassé pondéralement les deux autres fractions.

\section{Composition minérale de la litière (tableau I)}

Fraction "petits rameaux"

Les éléments analysés se classent ainsi, par ordre d'importance décroissante :

$\mathrm{Ca}>\mathrm{N}>\mathrm{K}-\mathrm{Mg}-\mathrm{Fe}>\mathrm{P}>\mathrm{Na}-\mathrm{Mn}$,

de façon identique à ce qui a été mis en évidence par les auteurs déjà cités, à l'exception du calcium qui est ici en concentration particulièrement élevée.

Les variations de concentration dans la litière sont fortes : elles sont à mettre en relation avec l'importance des précipita-

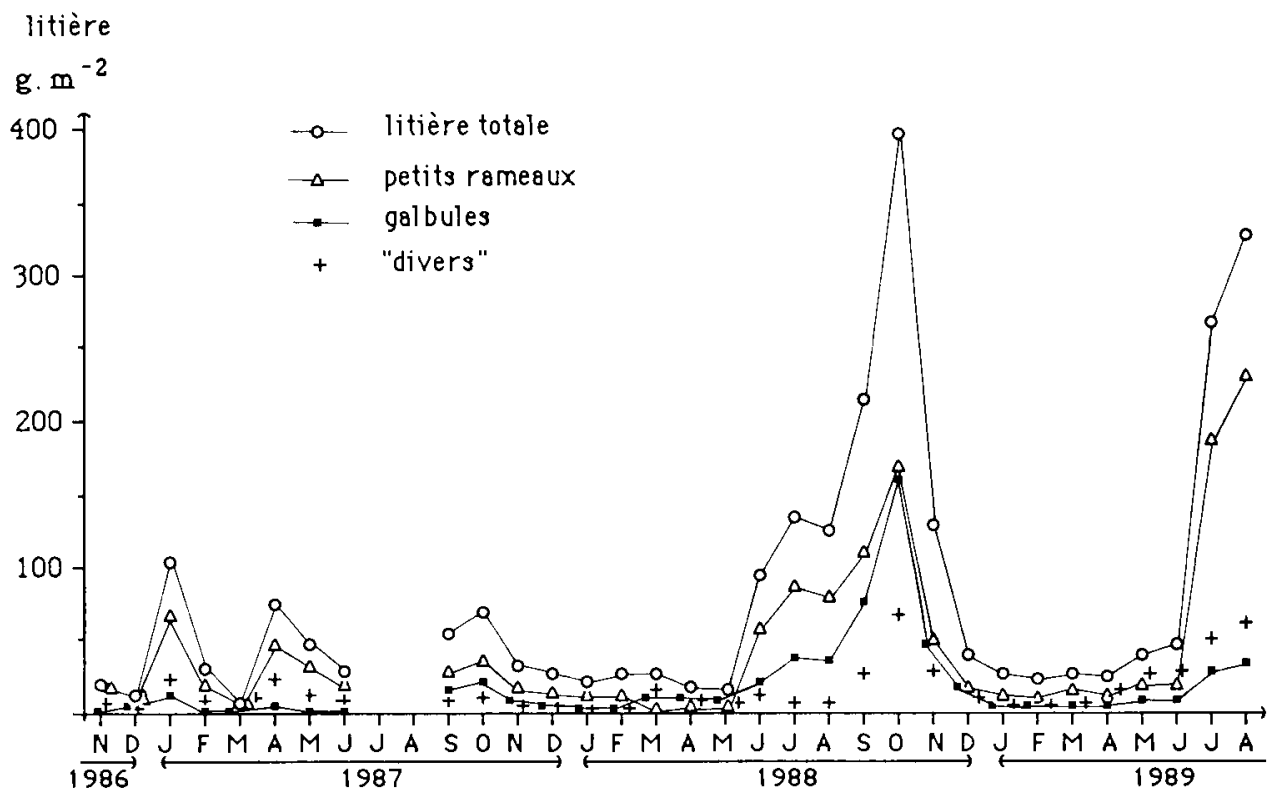

Fig 2. Rythme des retombées des différentes fractions de la litière sous le couvert d'un genévrier thurifère (parcelle de l'Oukaimeden, Haut Atlas, Maroc). 
Tableau I. Composition moyenne en éléments minéraux de la litière (fractions "petits rameaux", "divers" et "galbules") et des rameaux verts (\% de matière sèche \pm écart type).

\begin{tabular}{|c|c|c|c|c|c|c|c|c|}
\hline Éléments & $\mathrm{Ca}$ & $M g$ & $K$ & $\mathrm{Na}$ & $N$ & $P$ & $\mathrm{Fe}$ & $M n$ \\
\hline $\begin{array}{l}\text { Petits rameaux } \\
\text { (lit) }\end{array}$ & $\begin{array}{r}2,00 \\
\pm 0,51\end{array}$ & $\begin{array}{r}0,17 \\
\pm 0,04\end{array}$ & $\begin{array}{r}0,18 \\
\pm 0,05\end{array}$ & $\begin{array}{r}0,017 \\
\pm 0,009\end{array}$ & $\begin{array}{r}0,84 \\
\pm 0,16\end{array}$ & $\begin{array}{r}0,067 \\
\pm 0,020\end{array}$ & $\begin{array}{r}0,145 \\
\pm 0,060\end{array}$ & $\begin{array}{r}0,017 \\
\pm 0,004\end{array}$ \\
\hline $\begin{array}{l}\text { Divers } \\
\text { (lit) }\end{array}$ & $\begin{array}{r}2,28 \\
\pm 0,76\end{array}$ & $\begin{array}{r}0,15 \\
\pm 0,02\end{array}$ & $\begin{array}{r}0,16 \\
\pm 0,08\end{array}$ & $\begin{array}{r}0,029 \\
\pm 0,009\end{array}$ & $\begin{array}{r}0,82 \\
\pm 0.13\end{array}$ & $\begin{array}{r}0,063 \\
\pm 0,006\end{array}$ & $\begin{array}{r}0,444 \\
\pm 0,103\end{array}$ & $\begin{array}{r}0,024 \\
\pm 0,007\end{array}$ \\
\hline $\begin{array}{l}\text { Galbules } \\
\text { (lit) }\end{array}$ & $\begin{array}{r}0,55 \\
\pm 0,14\end{array}$ & $\begin{array}{r}0,10 \\
\pm 0,01\end{array}$ & $\begin{array}{r}0,49 \\
\pm 0,13\end{array}$ & $\begin{array}{r}0,028 \\
\pm 0,008\end{array}$ & $\begin{array}{r}1,06 \\
\pm 0,19\end{array}$ & $\begin{array}{r}0,083 \\
\pm 0,005\end{array}$ & $\begin{array}{r}0,071 \\
\pm 0,022\end{array}$ & $\begin{array}{r}0,009 \\
\pm 0,003\end{array}$ \\
\hline $\begin{array}{l}\text { Rameaux } \\
\text { verts }\end{array}$ & $\begin{array}{r}1,26 \\
\pm 0,30\end{array}$ & $\begin{array}{r}0,14 \\
\pm 0,02\end{array}$ & $\begin{array}{r}0,24 \\
\pm 0,07\end{array}$ & $\begin{array}{c}0,02 \\
\pm 0,007\end{array}$ & $\begin{aligned} & 1,030 \\
\pm & 0,16\end{aligned}$ & $\begin{array}{r}0,076 \\
\pm 0,020\end{array}$ & $\begin{array}{r}0,062 \\
\pm 0,016\end{array}$ & $\begin{array}{r}0,006 \\
\pm 0,001\end{array}$ \\
\hline
\end{tabular}

tions susceptibles de lixivier les différents éléments minéraux avant la chute des rameaux ou avant leur collecte.

\section{Autres fractions de la litière}

La concentration en fer est beaucoup plus forte dans la fraction «divers» que dans la fraction "petits rameaux $(0,44 \% \mathrm{~ms}$ contre $0,15 \%)$, celle du calcium également $(2,28 \%$ ms contre $2 \%)$, les chiffres sont comparables pour les autres éléments dosés. Les éléments se classent ainsi, par ordre d'importance décroissante :

$\mathrm{Ca}>>\mathrm{N}>\mathrm{Fe}>\mathrm{K}-\mathrm{Mg}>\mathrm{P}>\mathrm{Na}-\mathrm{Mn}$

Les galbules ont une concentration élevée en $N(1,06 \%)$ et en $\mathrm{K}(0,49 \%)$, enrichissant ainsi, lors de leur retombée, le sol en ces éléments el une teneur particulièrement faible en calcium $(0,55 \%)$. Les éléments s'ordonnent ainsi :

$\mathrm{N}>>\mathrm{Ca}-\mathrm{K}>\mathrm{Mg}>\mathrm{P}-\mathrm{Fe}-\mathrm{Mn}>\mathrm{Na}$

\section{Comparaison avec les rameaux verts}

L'ordre d'importance des éléments dosés dans les rameaux verts est le suivant, identique à celui indiqué par Rapp (1975) pour le pin d'Alep:

$\mathrm{Ca}>\mathrm{N}>\mathrm{K}-\mathrm{Mg}>\mathrm{P}>\mathrm{Fe} \mathrm{Na}>\mathrm{Mn}$

La concentration globale fluctue entre $2,43 \% \mathrm{~ms}$ (fin mai) et $3,51 \%$ (fin octobre) avec des nuances selon les éléments (Badri, 1990) :

- le calcium, constituant $40 \%$ de la somme totale des éléments dosés, présente la même évolution avec une concentration maximum $(1,63 \% \mathrm{~ms})$ fin octobre, une teneur minimum fin mai $(0,93 \%)$ et des fluctuations durant les mois d'été,

- la concentration en azote est relativement stable avec cependant une décroissance significative entre juillet et septembre,

- le potassium (K) montre 2 pics importants en septembre $(0,32 \% \mathrm{~ms})$ et octobre $(0,39 \%)$, les valeurs se stabilisent ensuite autour de $0,2 \%$,

- le magnésium, le manganèse et le phosphore sont en faibles concentrations, avec peu de fluctuations mais toujours un maximum enregistré fin octobre, 
- enfin, les concentrations en fer et sodium sont très variables, avec une augmentation en avril.

Ces résultats sont dans l'ensemble en accord avec ceux obtenus par divers auteurs (Guha et Mitchell, 1966; Fiedler et Hohne, 1965; Leroy, 1968).

Notons que les données ci-dessus concernent des moyennes relatives à un lot regroupant des individus mâles et des individus femelles. Dans les périodes hivernales (décembre en particulier), des différences assez nettes apparaissent en ce qui concerne le calcium entre, d'une part les rameaux prélevés sur individus mâles $(1,12 \% \mathrm{~ms})$ et, d'autre part, ceux prélevés sur individus femelles $(2,05 \%)$. Une étude plus fine sur l'évolution différentielle de la minéralomasse en fonction du sexe serait certainement intéressante à conduire.

Pour le $\mathrm{K}$, le $\mathrm{Na}$, le $\mathrm{N}$ et le $\mathrm{P}$ la teneur dans la litière est plus faible que dans les rameaux verts, avec une baisse respectivement de l'ordre de $25 \%, 20 \%, 18 \%$ et $12 \%$.
Par contre, le Ca est plus concentré dans la litière $(x 1,6)$, de même que le $\mathrm{Mn}$ $(x 2,8)$ et le $\mathrm{Fe}(x 2,3)$.

Seul le $M g$ ne présente pas de différence significative entre rameaux frais et rameaux issus des échantillons de litière.

Des résultats semblables ont été obtenus par Aussenac et al (1972) sur divers résineux (Pinus sylvestris, Picea abies et Abies grandis). De même Leroy (1968) apporte des conclusions analogues concernant $N, P$ et $K$ dans des litières de chênes pédonculés.

\section{Bilan annuel de la quantité d'éléments minéraux retournant au sol (tableau II)}

Pour la période d'étude, la quantité d'éléments minéraux restituée au sol par la litière est de $33,3 \mathrm{~g} \cdot \mathrm{m}^{2}$ de couvert.an ${ }^{-1}$ pour les éléments majeurs $(\mathrm{Ca}, \mathrm{Mg}, \mathrm{K}, \mathrm{Na}, \mathrm{N})$ et $1,77 \mathrm{~g}$ pour l'ensemble fer et manganèse. Ces résultats correspondent respectivement à $16 \mathrm{~g} \cdot \mathrm{m}^{-2} \cdot \mathrm{an}^{-1}$ et $0,85 \mathrm{~g} \cdot \mathrm{m}^{-2} \cdot \mathrm{an}^{-1}$ si l'on considère la surface totale de l'écosystème.

Tableau II. Apports annuels en éléments minéraux par la litière.

\begin{tabular}{lrrrrrrrrrrr}
\hline Éléments & $C a$ & $M g$ & $K$ & $N a$ & $N$ & $P$ & $\begin{array}{c}\text { Total } \\
\text { racro }\end{array}$ & $F e$ & $\begin{array}{c}\text { Mn } \\
\text { oligo }\end{array}$ \\
\hline $\begin{array}{l}\text { Apports 1987-1988 } \\
\text { (g.an-1.m-2 de couvert) }\end{array}$ & 8,82 & 0,89 & 1,55 & 0,28 & 5,29 & 0,51 & 17,34 & 1,31 & 0,13 & 1,44 \\
$\begin{array}{l}\text { Apports 1988-1989 } \\
\text { (g.an-1.m-2 de couvert) }\end{array}$ & 27,71 & 1,93 & 3,57 & 0,23 & 14,58 & 1,21 & 49,23 & 1,90 & 0,19 & 2,09 \\
$\begin{array}{l}\text { Moyenne } \\
\text { (g.an-1.m-2 de couvert) }\end{array}$ & 18,27 & 1,41 & 2,56 & 0,26 & 9,94 & 0,86 & 33,29 & 1,61 & 0,16 & 1,77 \\
$\begin{array}{l}\text { Moyenne } \\
\text { (g.an-1.m- } \mathrm{m}^{-2} \text { ) }\end{array}$ & 8,77 & 0,68 & 1,23 & 0,12 & 4,77 & 0,41 & 15,98 & 0,77 & 0,08 & 0,85 \\
\hline
\end{tabular}


Le calcium $\left(18,27\right.$ g. $\mathrm{m}^{-2}$ de couvert.an $\left.{ }^{-1}\right)$ et l'azote $\left(9,94\right.$ g.m ${ }^{-2}$ de couvert.an $\left.{ }^{-1}\right)$ représentent $81 \%$ de l'apport total sous l'arbre.

Les éléments se classent de la façon suivante :

$\mathrm{Ca}>\mathrm{N}>\mathrm{K}>\mathrm{Fe}-\mathrm{Mg}>\mathrm{P}>\mathrm{Na}-\mathrm{Mn}$,

comme pour la phytomasse aérienne, hormis le $\mathrm{K}$ qui est ici en concentration supérieure à $\mathrm{Fe}$ et $\mathrm{Mg}$.

Ces résultats sont identiques à ceux établis par Dannaoui (1981) et Rapp (1984) sur des pins méditerranéens mais différents de ceux d'Ulrich (1969) pour des forêts d'Europe centrale $(\mathrm{N}>\mathrm{Ca}>\mathrm{K}$ ). Courcoux (1982), dans une pinède landaise, signale une quantité de $\mathrm{N}$ et $\mathrm{Ca}$ du même ordre retournant au sol, avec de fortes concentrations en $\mathrm{Na}$ d'origine océanique.

\section{Apports d'éléments minéraux par la pluie et les pluviolessivats}

Parallèlement à la litière, la pluie et les pluviolessivats assurent au sol un gain en éléments minéraux et, à un degré moindre, en composés organiques.

Ces apports extérieurs ont des origines multiples : il s'agit d'une part d'éléments dissous dans les précipitations incidentes (dépôts humides), d'autres part d'éléments sous forme d'aérosols se déposant sur la végétation (dépôts secs), puis remobilisés et restitués au sol sous forme de pluviolessivats. II est certain que le feuillage joue dans ce cas un rôle de captage significatif; la rugosité de l'écorce et les diverses populations fongiques, algales ou lichéniques qui la colonisent favorisent également le piégeage des éléments sur les parties ligneuses.

À ces apports allochtones viennent s'ajouter des sécrétions minérales et orga- niques, que l'on retrouvera dans les pluviolessivats (Lossaint et Rapp, 1980).

\section{Précipitations incidentes, égouttement, écoulement et interception}

Les pourcentages moyens d'égouttement, d'écoulement et d'interception sont respectivement de $54 \%, 2,2 \%$ et $43,8 \%$, la valeur moyenne du seuil d'égouttement est de $3,89 \mathrm{~mm}$. On note une bonne corrélation entre précipitations et égouttement, avec une relation de la forme :

$E g(\mathrm{~mm}): 0,650 P_{i}(\mathrm{~mm})-2,530$

avec $n: 34$ et $R^{2}: 0,944$

Pour l'ensemble des événements recensés, ce sont dans les pluviomètres situés à la limite du couvert que les pourcentages d'égouttement sont les plus importants. Dans ces peuplements ouverts, la provenance des précipitations influe aussi sur le taux d'égouttement qui sont généralement supérieurs sous la portion de couronne exposée à cette pluie : par exemple, après une pluie venant de l'Est $(52 \mathrm{~mm}$, le 5 décembre 1988), le pourcentage d'égouttement a été de $77 \%$ du côté est et de $40 \%$ du côté ouest.

L'interception moyenne est particulièrement élevée, plus importante que celle déterminée pour d'autres peuplements de résineux en région méditerranéenne (Rapp et Romane, 1968; Ibrahim et al, 1982).

Les études menées par ailleurs par Gauquelin et Savoie (sous presse) montrent le rôle déterminant de cette forte interception sur l'évolution de l'humidité du sol sous le couvert des genévriers.

\section{Composition des eaux de pluie (tableau III)}

La charge des eaux de pluie en éléments minéraux est faible, avec une moyenne de 
Tableau III. Concentration moyenne en éléments minéraux (ppm \pm écarts types) dans la pluie incidente, les eaux d'égouttement et d'écoulement.

\begin{tabular}{|c|c|c|c|c|}
\hline \multirow{2}{*}{$\begin{array}{l}\text { Éléments } \\
\text { minéraux } \\
(p p m)\end{array}$} & \multicolumn{3}{|c|}{ Concentration (ppm) } & \multirow{2}{*}{$\begin{array}{c}\text { Rapport } \\
R=E g / P\end{array}$} \\
\hline & Pluies incidentes $(P)$ & Égouttement (Ag) & Écoulement (Ec) & \\
\hline $\mathrm{Ca}$ & $3,24 \pm 2,90$ & $13,80 \pm 10,04$ & $15,38 \pm 11,13$ & 4,3 \\
\hline $\mathrm{Mg}$ & $0,36 \pm 0,33$ & $3,00 \pm 1,97$ & $3,67 \pm 2,51$ & 8,3 \\
\hline $\mathrm{K}$ & $1,11 \pm 1,09$ & $12,50 \pm 15,61$ & $20,49 \pm 17,99$ & 11,3 \\
\hline $\mathrm{Na}$ & $1,35 \pm 1.45$ & $3.39 \pm 3,82$ & $4,90 \pm 4,71$ & 2,5 \\
\hline $\mathrm{P}$ & $0,54 \pm 0,69$ & $0,81 \pm 1,07$ & $4,30 \pm 8,41$ & 1,5 \\
\hline $\mathrm{Mn}$ & $0,03 \pm 0,03$ & $0,07 \pm 0,07$ & $0,11 \pm 0,08$ & 2,3 \\
\hline $\mathrm{NH}_{4}$ & $0,40 \pm 0,71$ & $0,98 \pm 2,36$ & $2,64 \pm 4,53$ & 2,5 \\
\hline $\mathrm{F}^{4}$ & $0,20 \pm 0,15$ & $0,41 \pm 1,02$ & $0,64 \pm 0,87$ & 2,1 \\
\hline $\mathrm{Cl}$ & $4,60 \pm 4,19$ & $11,34 \pm 10,77$ & $15,29 \pm 12,14$ & 2,5 \\
\hline $\mathrm{NO}_{3}$ & $0,45 \pm 0,32$ & $0,83 \pm 1,35$ & $1,38 \pm 1,39$ & 1,8 \\
\hline $\mathrm{SO}_{4}^{\circ}$ & $2,00 \pm 2,13$ & $4,60 \pm 5,32$ & $10,83 \pm 12,83$ & 2,3 \\
\hline Concentration totale & 14,54 & 55,10 & 86,41 & moyenne $=3,8$ \\
\hline
\end{tabular}

$14,5 \mathrm{mg} / \mathrm{l}$ et des valeurs mensuelles extrêmes de 5,6 mg/l (période neigeuse) et $58 \mathrm{mg} / \mathrm{l}$ (période estivale à faibles précipitations), la part des dépôts secs étant certainement importante dans ce dernier cas.

Le pH moyen est de 6,9 (écart-type : 0,54 ), avec 5,4 et 7,6 comme valeurs extrêmes, la variabilité est importante comme l'ont aussi montré d'autres auteurs en Grèce (Dikaiakos et al, 1990) ou en Israël (Mamane et Gottlieb, 1991).

Ces valeurs sont particulièrement élevées par rapport à celles obtenues dans les Pyrénées par exemple où une pollution acide diffuse a été mise en évidence $(\mathrm{pH}$ moyen : 4,04 dans le Luchonnais, Chéret, 1987; pH moyen : 4,47 à Lannemezan, Lefeivre, 1989).

Le tableau III donne pour chacun des éléments la concentration ionique moyenne calculée sur l'ensemble des éléments pluvieux ainsi que les concentrations minimales et maximales enregistrées.
Les éléments se classent de la façon suivante:

$\mathrm{Cl}>\mathrm{Ca}>\mathrm{SO}_{4}>\mathrm{Na}-\mathrm{N}\left(\mathrm{NO}_{3}+\mathrm{NH}_{4}\right)$

$-\mathrm{K}>\mathrm{P}>\mathrm{Mg}>\mathrm{F}-\mathrm{Mn}$

On remarquera à nouveau les variations importantes de concentrations pour certains ions (fortes valeurs des écarts types), rejoignant ainsi les observations de $\mathrm{Ma}$ mane et Gottliev en Isräel (loc cit) ou de Ovalle et al (1991) au Brésil.

Les valeurs de $\mathrm{Ca}, \mathrm{K}, \mathrm{Cl}$ et $\mathrm{Na}$ sont nettement plus élevées que celles mentionnées par Granat (1972), Dambrine et Prevosto (1989) en France ou par Santa Regina et Gallardo (1989) en Espagne (l'étude n'a cependant porté dans ce dernier cas que sur une période hivernale). Elles sont du même ordre de grandeur que celles de Mamane et Gottlieb en Israël (loc cit). Pour ces derniers auteurs, les dépôts importants de calcium en particulier sont apportés lors de tempêtes mobilisant en quantité des particules terrigènes basiques. 
Les concentrations en nitrates et sulfates, liées pour une part à la pollution mais aussi à une possible origine marine pour ces derniers, sont comparables à celles indiquées par les auteurs cités cidessus, de même que les concentrations en ammonium et magnésium.

\section{Composition chimique des pluviolessivats (tableau III)}

\section{Eaux d'égouttement}

La charge moyenne des eaux d'égouttement, ou pluviolessivats, est de $55,10 \mathrm{mg} /$ I, soit 3,8 fois celle des pluies incidentes. Cette augmentation est cependant variable selon les ions : la concentration en $\mathrm{K}$ est multipliée sous couvert par 11,3 , celle du Ca par 4,3 et de $\mathrm{NO}_{3}$ par seulement 1,8 . L'ordre des éléments est alors le suivant:

$\mathrm{Ca}>\mathrm{K}>\mathrm{Cl}>\mathrm{SO}_{4}>\mathrm{Na}-\mathrm{Mg}>\mathrm{N}>\mathrm{P}>$ $\mathrm{F}>\mathrm{Mn}$

le pH varie entre 6,2 et 7,95 , avec une valeur moyenne de 7,25 (écart type : 0,43), plus élevée que celle de la pluie incidente.

Trois types de processus peuvent être à l'origine des modifications de concentrations entre pluies et pluviolessivats :

- simple concentration par évaporation de l'eau interceptée : on suppose alors que le feuillage traversé n'agit en aucune façon sur la composition chimique de l'eau,

- lessivage de dépôts secs ou occultes (déposés en dehors des périodes de pluie), et/ou d'éléments minéraux secrétés par le feuillage : il y a augmentation de la charge minérale de l'eau,

- rétention d'éléments minéraux par le feuillage : la concentration est ici au contraire diminuée.

Afin de discuter nos résultats dans ce sens, 2 rapports ont été calculés :
- le rapport (R) des concentrations moyennes des eaux d'égouttement à celles des précipitations incidentes, pour chacun des éléments (tableau VI),

- le coefficient moyen de concentration par interception, défini par Wedraogo-Dumazet (1983) : $C=1 / 1-l$ avec $I=$ interception moyenne du peuplement en $\%$.

Pour la parcelle étudiée, $I=44 \%$, d'où $C=1,79$.

Pour la majorité des éléments chimiques, nous avons $R>C$ : cette relation signifie que leur charge réelle dans les pluviolessivats est supérieure à leur charge théorique résultant du seul phénomène de concentration par évaporation de l'eau interceptée. II y a donc eu incorporation dans les eaux recueillies d'éléments issus de dépôts secs etou secrétés par le feuillage. Le rôle des dépôts secs semble ici prédominer : on note par exemple une très forte augmentation de concentration en $\mathrm{Ca}(x 10), \mathrm{Mg}\left(x_{10}\right), \mathrm{K}(\mathrm{x} 50)$ et $\mathrm{Na}(\mathrm{x} 3)$ en septembre 1988, traduisant le lavage par les premières pluies de l'automne des éléments minéraux déposés durant l'été sur les surfaces foliaires.

Pour les nitrates, les coefficients $R$ et $C$ sont comparables : on peut supposer alors que les phénomènes d'incorporation de dépôts secs et de rétention par le feuillage s'équilibrent.

Pour $\mathrm{P}$, nous avons $R<C$, la rétention est sans doute ici le phénomène dominant.

\section{Écoulement}

La concentration moyenne en éléments minéraux des eaux d'écoulement est forte, $(86,41 \mathrm{ppm})$, soit près de 6 fois supérieure à la teneur des eaux de pluie et 1,6 fois à celle des eaux d'égouttement.

Le $\mathrm{pH}$ moyen est de 7,52 (écart type $=$ $0,46)$.

Il faut rappeler ici que les troncs des genévriers thurifères ont une écorce particu- 
Tableau IV. Apports annuels d'éléments minéraux par les égouttements, les écoulements et la pluie incidente ( $\mathrm{g} \cdot \mathrm{an}^{-1} \cdot \mathrm{m}^{-2}$ de couvert) : détermination des apports liés au couvert.

\begin{tabular}{|c|c|c|c|c|c|c|c|c|}
\hline & \multirow{2}{*}{$\begin{array}{l}\text { Hauteur } \\
\text { d'eau } \\
(\mathrm{mm})\end{array}$} & \multicolumn{7}{|c|}{ Éléments minéraux } \\
\hline & & $\mathrm{Ca}$ & $M g$ & $K$ & $\mathrm{Na}$ & $M n$ & $N$ & $P$ \\
\hline Apports par égouttement (1) & 566,1 & 2,69 & 0,64 & 2,35 & 0,65 & 0,06 & 1,34 & 0,48 \\
\hline Apports par écoulement (2) & 22,6 & 0,14 & 0,04 & 0,17 & 0,05 & 0,002 & 0,12 & 0,04 \\
\hline $\begin{array}{l}\text { Apports sous couvert } \\
(3)=(1+2)\end{array}$ & & 2,83 & 0,68 & 2,52 & 0,70 & 0,0062 & 1,46 & 0,52 \\
\hline $\begin{array}{l}\text { Apports hors couvert (4) } \\
\text { (pluie incidente) }\end{array}$ & 1052,1 & 1,59 & 0,18 & 0,49 & 0,66 & 0,03 & 0,47 & 0,81 \\
\hline $\begin{array}{l}\text { Apports liés au couvert } 5=(3-4) \\
\text { (Facteurs de concentration) }\end{array}$ & & $\begin{array}{c}1,24 \\
(\times 1,8)\end{array}$ & $\begin{array}{l}0,50 \\
(\times 3,8)\end{array}$ & $\begin{array}{l}2,03 \\
(\times 5,1)\end{array}$ & $\begin{array}{r}0,004 \\
(\times 1,1)\end{array}$ & $\begin{array}{c}0,032 \\
(\times 2)\end{array}$ & $\begin{array}{l}0,99 \\
(\times 3)\end{array}$ & $\begin{array}{l}-0,29 \\
(\times 0,6)\end{array}$ \\
\hline
\end{tabular}

lièrement épaisse et rugueuse qui constitue un piège efficace pour les particules minérales susceptibles ensuite d'être lessivées.

L'importance relative des éléments dans les eaux d'écoulement est la suivante:

$\mathrm{K}>\mathrm{Ca}-\mathrm{Cl}>\mathrm{N}-\mathrm{SO}_{4}>\mathrm{P}-\mathrm{Na}>\mathrm{MgF}>\mathrm{Mn}$

\section{Bilan annuel de la quantité d'éléments minéraux apportés au sol par les pluies et les pluviolessivats (tableau IV)}

La quantité annuelle d'éléments minéraux véhiculés par les eaux et arrivant au sol est nettement supérieure sous couvert, hormis pour $\mathrm{Na}$ dont les apports sont peu modifiés et pour le $P$ qui est en partie retenu par le feuillage.

On voit par exemple que $2,52 \mathrm{~g}$ de K. $\mathrm{m}^{-2}$ sont amenés annuellement au sol sous couvert par les pluviolessivats alors que hors couvert les pluies incidentes n'en apportent que $0,49 \mathrm{~g}$ : pour cet élément la présence du feuillage multiplie par 5,1 les apports par le vecteur eau.
Les apports sont multipliés par 3,8 sous couvert pour le $\mathrm{Mg}(0,68 \mathrm{~g}$ contre $0,18 \mathrm{~g})$ et par 3 pour $\mathrm{N}(1,46 \mathrm{~g}$ contre $0,47 \mathrm{~g})$.

Pour le calcium et le manganèse, les apports sont sensiblement doublés (respectivement $2,83 \mathrm{~g}$ et $0,062 \mathrm{~g}$ contre 1,59 $\mathrm{g}$ et $0,03 \mathrm{~g}$ ).

Dans ces pluviolessivats, les eaux d'écoulement ne représentent qu'une faible partie des apports, moins de $10 \%$, l'essentiel étant apporté par les eaux d'égouttement.

\section{DISCUSSION}

Sous le couvert du genévrier, 2 vecteurs enrichissent donc le sol en éléments minéraux,

- les piuviolessivats qui contiennent une part d'éléments déjà présents dans les eaux de pluie et une autre provenant du lavage du feuillage : ces apports, en termes quantitatifs (g.an-1. $\mathrm{m}^{-2}$ de couvert), sont précisés dans le tableau IV, 
Tableau V. Pourcentage relatif des éléments apportés au sol sous couvert par la litièra et par les pluviolessivats $\left(\mathrm{g} \cdot \mathrm{an}^{-1} \cdot \mathrm{m}^{-2}\right.$ de couvert).

\begin{tabular}{lcccccccccc}
\hline $\begin{array}{l}\text { Eléments } \\
\text { minéraux }\end{array}$ & $C a$ & $M g$ & $K$ & $N a$ & $N$ & $P$ & $\begin{array}{r}\text { Total } \\
\text { macro }\end{array}$ & Fe & Mn \\
\hline Litière \% & 86,6 & 67,5 & 50,4 & 27,1 & 87,2 & 62,3 & 79,3 & $\approx 100$ & 72,7 \\
Pluviolessivats \% & 13,4 & 32,5 & 49,6 & 72,9 & 12,8 & 37,7 & 20,7 & - & 27,3 \\
\hline
\end{tabular}

- la litière : les apports déjà exprimés en termes quantitatifs (tableau II) sont comparés à ceux provenant des eaux recueillies sous couvert dans le tableau $V$.

Selon les éléments, la part prise par l'un ou l'autre de ces compartiments est variable:

- les apports sont équilibrés pour le $\mathrm{K}$ entre pluviolessivats et litière (respectivement $2,52 \mathrm{~g}$ et $2,56 \mathrm{~g}$ soit $49,6 \%$ et $50,6 \%)$,

- le N $(87,2 \%)$, le Ca $(86,6 \%)$, le $\mathrm{Mn}$ $(72,7 \%)$, le $\mathrm{Mg}(67,5 \%)$ et le $\mathrm{P}(62,3 \%)$ proviennent majoritairement de la litière, mais les proportions issues des eaux recueillies sous couvert ne sont jamais négligeables;

- le $\mathrm{Na}$ provient majoritairement des pluviolessivats $(72,9 \%), 27,1 \%$ seulement étant apportés par la litière.

La litière a donc souvent un rôle essentiel d'approvisionnement en éléments minéraux des horizons superficiels du sol.

Elle est en effet produite en quantité importante (1 100 g.an-1. $\mathrm{m}^{-2}$ de couvert) malgré les conditions climatiques sévères et malgré la vitesse de croissance très lente du genévrier. Particulièrement riche en calcium et en azote, elle est à l'origine de l'existence sous le couvert du thurifère d'un horizon humifère épais aux caractéristiques ( $\mathrm{pH}: 7,3 ; \mathrm{C} / \mathrm{N}: 20,7$; taux de saturation du complexe absorbant entre 70 et $100 \%$ ) peu influencées par le matériau parental acide.
Ces apports via la litière constituent un recyclage d'éléments puisés sous la couronne même de l'arbre mais aussi hors couvert du fait de l'extension du système racinaire. II se produit donc dans ces milieux ouverts un phénomène de concentration des éléments minéraux sous le couvert des arbres qui exploitent l'ensemble des potentialités nutritives du milieu.

Les apports d'éléments par les pluviolessivats, en proportion généralement plus faible mais directement absorbables par les racines, correspondent par contre, au moins en partie, à un gain net pour l'écosystème (éléments dissous dans les précipitations incidentes et dépôts secs).

Cependant, la richesse en cations de ces pluviolessivats est en moyenne supérieure à celle relevée par différents auteurs dans des écosystèmes méditerranéens. On peut penser que dans les écosystèmes constitués par des arbres isolés le phénomène de filtration des aérosols par le feuillage se manifeste avec une intensité supérieure. Ainsi, dans les formations ouvertes à chênes verts en Espagne, les facteurs d'enrichissement observés sous les arbres sont très supérieurs à ceux généralement données pour des formations fermées équivalentes (Escudero, 1985).

En tout état de cause, la quantité d'éléments minéraux mise en mouvement par l'intermédiaire des pluviolessivats et de la litière s'avère importante; strictement localisés au couvert de l'arbre, les apports par- 
ticipent au maintien des conditions édaphiques, floristiques et microclimatiques particulières à ce secteur protégé par la couronne des arbres.

If convient de rappeler enfin que ce couvert constitue le lieu quasi exclusif des rares germinations de genévrier.

Si dans la thuriféraie étudiée ici ces conditions de couvert se maintiennent, il n'en est pas de même dans la majorité des peuplements, soumis à une dégradation anthropique intense. La mutilation des houppiers entraîne ainsi dans ces milieux 2 types de conséquences néfastes au maintien et au développement de la thuriféraie,

- la diminution des apports qui en résulte entraîne un amenuisement de l'horizon organique; les processus pédogénétiques sont entravés, les phénomènes érosifs se développent sous l'impact direct des précipitations qui ne sont plus interceptées par le couvert des arbres,

- le processus de régénération est contrarié : les germinations ne se développent plus sur un substrat qui leur est désormais hostile.

\section{RÉFÉRENCES}

Achaal A, Avabli O, Barbero M, M'Hirit O, Peyre C, Quézel P, Rivas-Martinez S (1980) À propos de la valeur bioclimatique et dynamique de quelques essences forestières du Maroc. Oecol Medit V, 211-249

Antiwill PM (1968) The loss of element from decomposing litter. Ecology 49, 142-145

Aussenac G (1979) Production de litière dans 4 peuplements de douglas de l'Est de la France. Rev For Fr XXXI, 1 : 15-19

Aussenac G, Bonneau M, Le Tacon F (1972) Restitution des éléments minéraux au sol par l'intermédiaire de la litière et des précipitations dans quatre peuplements forestiers de l'Est de la France. Oecol Plant 7, 1-21

Badri W (1990) Cycle hydrologique et biogéochimique et influence du couvert sur la strate herbacée dans un peuplement à Genévrier thurifère du Haut Atlas de Marrakech (Maroc). Thèse Université Cadi Ayyad, Marrakech, $167 \mathrm{p}+$ annexes

Barbero M, Quézel P (1984) Caractérisation bioclimatique des étages de végétation forestière sur le pourtour méditerranéen. Aspects méthodologiques posés par la zonation. Doc Ecol Pyr III-IV, 49-56

Bray JR, Gorham E (1964) Litter production in forests of the world. Adv Ecol Res 2 : 101157

Cabannetes A (1979) Croissance, biomasse et productivité de Pinus pinea $L$ en petite $\mathrm{Ca}$ margue. Thèse doct $3^{e}$ cycle, USTL, Montpellier, $175 \mathrm{p}$

Cheret V (1987) La sapinière du Luchonnais (Pyrénées Hautes-Garonnaises) : Étude phytoécologique; recherches sur le phénomène de dépérissement forestier. Thèse Doctorat Université P Sabatier, Toulouse, 287 p

Courcoux $P$ (1982) Approche du cycle biologique d'un écosystème à Pin maritime (Pinus pinaster Aït) en lande humide. DEA Université de Bordeaux III, $45 p+9$ annexes

Dambrine $E$, Prevosto $B$ (1989) Flux des éléments minéraux dans un écosystème forestier d'altitude soumis à la pollution atmosphérique. Relation avec le dépérissement. Journées de travail DEFORPA, INRA Nancy, vol 4, 82-142

Dannaoui S (1981) Production de litière et restitution au sol d'éléments biogènes dans des peuplements méditerranéens de Pinus pinea $L$ et Pinus brutia Ten. Ecol Medit VII (2), 13-25

Dikaiakos JG, Tsitouris CG, Siskos PA, Melissos DA, Nastos $P$ (1990) Rainwater composition in Athens, Greece. Atmos Environ 24B, 171-176

Emberger $L$ (1939) Aperçu général de la végétation du Maroc. Commentaire de la carte phytogéographique du Maroc au 1/500 000. Veröff Geobot Inst Rübel in Zurich 14, 40-157

Escudero A (1985) Efectos de arboles aislados sobre las propriedas quimicas del suelo. Rev Ecol Biol Sol 22 (2), 149-159

Escudero A, Garcia B, Gomez JM, Luis E (1985) The nutrient cycling in Quercus rotundifolia and Quercus pyrenaica ecosystems ("dehesas") of Spain. Acta Oecol Oecol Plant $6(20) n^{\circ} 1,73-86$ 
Fiedler HJ, Hohne H (1965) Vorkommen und gehalt der makronährstoffe in Waldbiimen. Wisshaftliche Zertschrift des Technischen Universität Dresden 1(4)

Fromard F, Gauquelin T (1992) Les formations à genévrier thurifère des montagnes marocaines : actions de recherche et de conservation sur un milieu et une espèce en régression. Unasylva, FAO (sous presse)

Gauquelin T (1988) Dynamique de la végétation et des formations superficielles dans les montagnes du bassin occidental de la Méditerranée : étude des formations à genévrier thurifère et à xérophytes épineuses en coussinet des Atlas marocains. Thèse doct État, Univ P Sabatier, Toulouse : Tome I : 148 p; Tome II : tableaux et figures

Gauquelin T (1989) Les peuplements d'altitude à genévrier thurifère des Atlas marocains : structure et dynamique. Acta Biol Mont (IX), 209-216

Gauquelin T, Dagnac $J$ (1988) Caractéristiques édaphiques des groupements à Genévrier thurifère des Atlas marocains : étude des niveaux superficiels des sols sous couvert et hors couvert. Oecol Medit XIV (3/4), 43-46

Gauquelin T, Savoie JM Évolution de l'humidité du sol en relation avec le couvert arboré dans un écosystème à Juniperus thurifera $\mathrm{L}$ du Haut Atlas de Marrakech (Maroc). Actes du colloque "Écologie et Biogéographie alpines" La Thuile, Sept 90 (sous presse)

Granat $L$ (1972) On the relation between $\mathrm{pH}$ and the chemical composition in atmospheric precipitation. Tellus 24, 551-560

Guha M, Mitchel RLV (1966) The trace and major element composition of the leaves of some deciduous trees. Seasonal changes. Plant Soil 24, 90-112

Ibrahim M, Rapp M, Lossaint P (1982) Économie de l'eau d'un écosystème à pin pignon (Pinus pinea L) du littoral méditerranéen. Ann Sci For 39, 289-306

Lefeivre $B$ (1989) Composition chimique et acidité des précipitations recueillies dans les pyrénées centrales : relations entre les situations météorologiques et les processus d'acidification. Mémoire de DEA, Université P Sabatier, Toulouse $68 \mathrm{p}$

Leonardi S, Rapp M, La Rosa V (1988) Répartition et dynamique de la matière organique dans une forêt de Pinus laricio Poir. Oecol Medit XIV (3/4), 17-31

Lepoutre B (1961) Recherches sur les conditions édaphiques de régénération des Cédraies marocaines. Ann Rech For Maroc 6(2), Rabat

Leroy $P$ (1968) Variations saisonnières des teneurs en eau et éléments minéraux des feuilles de chênes (Quercus pedunculata Ehrh). Ann Sci For 25, 83-117

Lossaint $P$, Rapp M (1980) La forêt méditerranéenne de chêne vert (Quercus ilex). In : Problèmes d'écosystèmes terrestres, Lamotte et Bourlière. Masson éd, 129-185

Mamane $Y$, Gottlieb $J(1991)$ Chemistry of precipitation in Haifa, Israël, 1981-1989. In: Acid deposition. Origins, impacts and abatement strategies (James WS Longhurst, ed) Springer Verlag, 3-11

Morard P, Gullo JH (1970) Minéralisation de tissus végétaux en vue du dosage de $\mathrm{P}, \mathrm{K}, \mathrm{Ca}$, $\mathrm{Mg}$ et Na. Ann Agr 21(2), 229-236

Ovalle Arc, Silva Filho EV (1992) Acid precipitation research in Brazil: a short review. In : Acid deposition. Origins, impacts and abatement strategies (James WS, Longhurst, ed) Springer Verlag, 51-59

Peyre C (1975) Contribution à l'étude de la végétation du Moyen Atlas oriental : le versant sud oriental du massif du Bou Iblane. CNRS, Trav RCP 249, III, 97-142

Quezel P (1957) Peuplement végétal des hautes montagnes de l'Afrique du Nord. Encycl Biol Ecol Paul Lechevalier, Paris, 1-463

Rapp M (1967) Production de litière et apport d'éléments minéraux et d'azote dans un bois de pin d'Alep (Pinus halepensis Mili). Oecol Plant 2, 325-338

Rapp M (1969) Apport d'éléments minéraux au sol par les eaux de pluviolessivats sous des peuplements de Quercus ilex L, Quercus lanuginosa Lam et Pinus halepensis Mill. Oecol Plant 4, 71-92

Rapp M (1975) Le cycle biogéochimique dans un bois de pin d'Alep. In : Ecol For. Gauthiers-Villars, 75-97

Rapp M (1984) The monthly cycling of nutrients in a stand of Pinus pinea L, Dept Ecol Environ Res 13, 261-291

Rapp M, Romane F (1968) Contribution à l'étude du bilan de l'eau dans les écosystèmes méditerranéens. 1. Égouttement des 
précipitations sous des peuplements de Quercus ilex $L$ et de Pinus halepensis Mill. Oecol Plant 3, 271-284

Santa Regina I, Gallardo JF (1989) Biogeochemical cycles in forests of the Sierra de Béjar (Salamanca, Spain): return of bioelements in rainfall. Acta Oecol Oecol Plant 10, 433438

Selmi R (1985) Différenciation des sols et fonctionnement des écosystèmes forestiers sur grés numidien de Kroumirie (Tunisie). Écologie de la suberaie zeenaie.
Thèse de doctorat, Université de Nancy I, France, $229 \mathrm{p}$

Ulrich B (1969) Recherche sur le cycle des éléments nutritifs dans les forêts d'Europe Centrale. Act Coll Bruxelles (Ecol et Cons 4), UNESCO 1971, 501-506

Wedraogo Dumazet B (1983) Modification de la charge chimique des eaux au cours du transit à travers 3 écosystèmes distincts du Mont-Lozère (Hêtraie, pessière, pelouse). Thèse doct Ecol Appl, Université d'Orléans, France, $147 \mathrm{p}$ 